\title{
Análise espectral do sinal eletromiográfico do músculo eretor da espinha obtido do teste de Sorensen
}

\author{
Spectral analysis of the electromyographic signal of the erector spinae muscle \\ obtained from Sorensen test
}

Fernando Sérgio Silva Barbosa ${ }^{[a]}$, Camila Cristina Rodeline Almeida ${ }^{[b]}$, Mauro Gonçalves ${ }^{[\mathrm{c}]}$

[a] Professor Mestre, chefe do Departamento Interdisciplinar de Tecnologia e Ciências (DINTEC) e vice-chefe do Departamento de Ciências da Educação (DECED) da Universidade Federal de Rondônia (UNIR), Rondônia, RO - Brasil, e-mail: fernandossb@hotmail.com

[b] Fisioterapeuta e membro do Laboratório de Biomecânica do Departamento de Fisioterapia do Centro Universitário Luterano de Ji-Paraná (CEULJI/ULBRA), Ji-Paraná, RO - Brasil.

[c] Professor Doutor e coordenador do Laboratório de Biomecância do Departamento de Educação Física da Universidade Estadual Paulista (UNESP), câmpus de Rio Claro, SP - Brasil.

\section{Resumo}

Introdução: A fadigabilidade excessiva dos músculos lombares é um achado comum em pacientes portadores de dor lombar. Por isso, a avaliação da resistência isométrica desses músculos tem sido recomendada nessa população. Objetivo: $\mathrm{O}$ objetivo deste estudo foi identificar e analisar objetivamente o comportamento da fadiga do músculo eretor da espinha em um teste de resistência isométrica realizado até a exaustão. Metodologia: Nove sujeitos saudáveis realizaram o teste de Sorensen modificado com contrações em intensidades correspondentes a 5\%,10\%,15\% e 20\% da contração voluntária máxima. A fadiga muscular foi identificada pela análise do comportamento da frequência mediana (FM) em função do tempo. Resultados: O tempo de resistência isométrica foi inversamente correlacionado com a intensidade da contração. Contudo, a intensidade da contração não demonstrou efeito sobre a taxa de declínio da FM. A fadiga muscular foi significante em todas as porções do músculo eretor da espinha. Comparações entre os músculos eretor da espinha direito e esquerdo não revelaram diferenças significantes, enquanto que comparações entre porções do músculo eretor da espinha localizadas em diferentes níveis lombares revelaram maiores níveis de fadiga em L4-L5 bilateralmente. Conclusão: A análise espectral do sinal eletromiográfico foi eficaz para idenficação da fadiga do músculo eretor da espinha. Também foi possível identificar diferenças funcionais entre as diferentes porções desse músculo. O conhecimento 
dessas particularidades permite intervir de forma mais específica na prevenção e reabilitação dos distúrbios da coluna lombar.

Palavras-chave: Coluna vertebral. Eletromiografia. Contração isométrica. Fadiga muscular.

\begin{abstract}
Introduction: Excessive fatigability of the low back muscles is a common finding in low back pain patients. Therefore, the assessment of the isometric endurance of these muscles has been recommended in this population. Objective: The purpose of this study was to identify and to analyze objectively the behavior of the erector spinae muscle fatigue in an isometric endurance test performed until exhaustion. Method: Nine healthy subjects performed a modified Sorensen test with contractions of 5\%,10\%,15\%, and 20\% of the maximal voluntary contraction. The muscle fatigue was identified for the analysis of the behavior of the median frequency (MF) over time. The endurance time was inversely correlated with the contraction intensity. However, the contraction intensity did not show effect on the rate of decline of the MF. The muscle fatigue was significant in all the portions of the erector spinae muscle. Results: Comparisons between the erector spinae muscles from right and left side did not show significant differences, while that the comparisons between the portions of the erector spinae muscle localized at different vertebral levels showed higher fatigue levels at L4-L5 bilaterally. Conclusion: Thus, the spectral analysis of the electromyographic signal was effective for the identification of the erector spinae muscle fatigue. Also, it was possible to identify functional differences between the different portions of this muscle. The knowledge of these particularities allows managing in a more specific way the prevention e rehabilitation of the low back troubles.
\end{abstract}

Keywords: Spine. Electromyography. Isometric contraction. Muscle fatigue.

\title{
Introdução
}

Os dados estatísticos mais recentes fornecidos pela Previdência Social demonstram que dos 653.090 acidentes de trabalho registrados no ano de 2008, 23.888 foram relacionados com a coluna vertebral. Aproximadamente 50\% desses acidentes foram cadastrados no Instituto Nacional do Seguro Social (INSS) como dor nesse segmento corporal. Esses números contribuíram com a concessão de mais de 2.900.000 benefícios, os quais somaram mais de $\mathrm{R} \$ 1.300 .000$ em pagamentos de benefícios (1).

Números relevantes como esses destacam a importância do desenvolvimento de instrumentos e protocolos de avaliação da capacidade funcional de músculos lombares. A análise da força $(2,3)$ e da resistência isométrica (4-6) desses músculos são métodos comumente utilizados para estabelecer prognósticos para o desenvolvimento da dor lombar.

Contudo, a avaliação de um músculo por meio dos clássicos testes manuais de força ou por meio de testes nos quais se avalia a resistência de um músculo por meio da manutenção pelo maior tempo possível de uma contração isométrica e o registro deste tempo pelo avaliador (tempo de resistência isométrica [TRI]), tem sido questionada. Esse questionamento se deve ao fato de ambos os métodos de avaliação sofrerem grande influência de fatores subjetivos como motivação e dor $(7,8)$. Por essa razão, esses parâmetros, denominados de mecânicos, têm sido considerados como pouco confiáveis para realmente fornecer informações a respeito do estado de um músculo, ao contrário do que ocorre com a utilização de parâmetros denominados de fisiológicos, os quais podem ser obtidos com o uso da eletromiografia.

Nesse sentido, para avaliar o estado de um músculo e, em particular a fadiga muscular, sem que haja influência desses fatores previamente descritos, destacam-se as técnicas e parâmetros obtidos a partir da eletromiografia. A partir da eletromiografia é possível a avaliação da função muscular por meio de análises realizadas no domínio do tempo $(9,10)$, por meio das quais se pode verificar mudanças na amplitude dos disparos das unidades motoras, e análises no domínio das frequências (11, 12), em que é possível verificar 
mudanças nas frequências de disparos dessas unidades motoras, possibilitando uma avaliação muito mais confiável do estado de um músculo.

Esse instrumento biomecânico tem sido utilizado em testes específicos realizados em diferentes posturas. Entre eles, destaca-se o teste de Sorensen, no qual o sujeito realiza a extensão isométrica do tronco em decúbito ventral $(13,14)$. Entretanto, o comportamento dos músculos lombares durante a realização desse teste é avaliado considerando apenas a contração em intensidade suficiente para a manutenção do tronco suspenso $(7,11,15,16)$, desconsiderando-se os efeitos de contrações de maior intensidade comumente realizadas durante o dia em resposta às atividades da vida diária (AVD), trabalho e esporte.

Com base nessas informações, o objetivo deste estudo foi avaliar o comportamento espectral do sinal eletromiográfico (EMG) do músculo eretor da espinha durante teste isométrico realizado em intensidades correspondentes a porcentagens da contração voluntária máxima (CVM).

\section{Materiais e métodos}

\section{Sujeitos}

Nove estudantes universitários do gênero masculino (idade: $20,4 \pm 1,3$ anos; massa corporal: $68,7 \pm$ 7,4 kg; altura: $1,7 \pm 0,06 \mathrm{~m}$ ), sem história de patologias músculo-esqueléticas na coluna vertebral e sem história de dor lombar nas quatro semanas que antecederam o estudo (17) participaram do presente estudo. Os sujeitos foram selecionados aleatoriamente mediante carta-convite após constatação de características físicas aparentemente similares ao restante da amostra.

O estudo foi aprovado pelo Comitê de Ética local, de acordo com a Resolução 196/96 do Conselho Nacional de Saúde. Todos os voluntários assinaram um termo de consentimento livre e esclarecido contendo informações acerca dos procedimentos aos quais seriam submetidos e que garantiam sua privacidade.

\section{Posicionamento dos sujeitos e equipamentos}

Para a realização da CVM e das contrações submáximas, os sujeitos foram posicionados em decúbito ventral sobre uma mesa de testes (Figura 1). A pelve e os membros inferiores foram fixados à base da mesa de testes por meio de três cintos de couro.

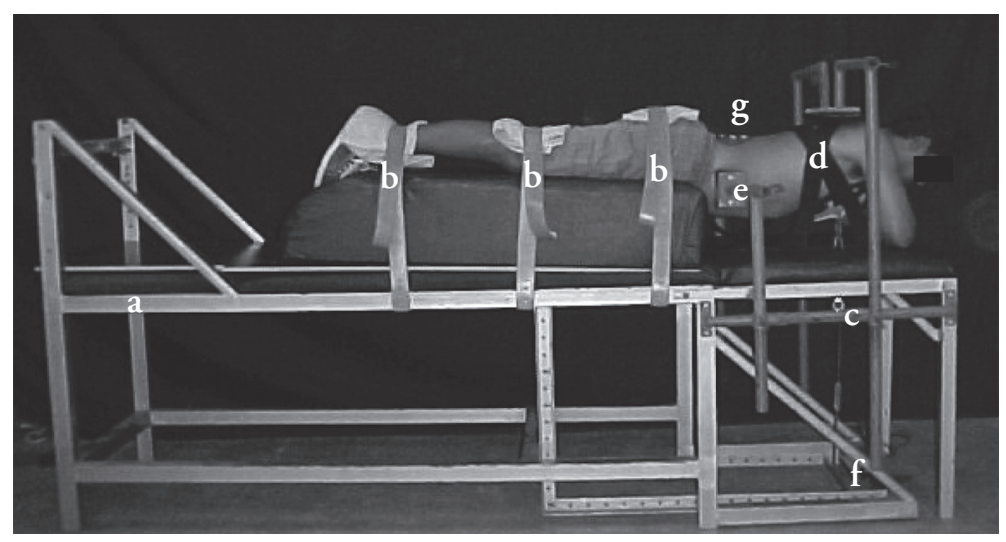

Figura 1 - Postura dos sujeitos e equipamentos utilizados para a CVM e contrações submáximas. a: mesa de testes; b: cintos de couro; c: célula de carga; d: colete; e: limitadores de movimento; f: indicador digital; g: eletrodos 
O movimento realizado em ambos os testes foi a extensão isométrica do tronco em decúbito ventral, com os membros superiores cruzados em frente ao tórax. Esse movimento foi realizado por meio da tração em uma célula de carga (MM $100 \mathrm{~kg}$, $\operatorname{Kratos}^{\circledR}$ Dinamômetros LTDA.), do tipo strain gage, acoplada perpendicularmente a um colete utilizado pelos sujeitos e à base da mesa de testes. Limitadores de movimento posicionados bilateralmente sobre as escápulas e lateralmente no tronco evitaram a ocorrência da rotação e inclinação lateral do tronco, respectivamente. Para que os sujeitos pudessem controlar a intensidade da contração durante as contrações submáximas, um indicador digital (IK 14A, Kratos ${ }^{\circledR}$ Dinamômetros LTDA.) foi posicionado no solo abaixo dos sujeitos.

\section{CVM e contrações submáximas}

Inicialmente foi determinada a CVM de cada sujeito. Esse procedimento foi realizado em três dias com intervalo mínimo de 24 e máximo de 48 horas entre cada dia de teste (18). Em cada um dos dias, os sujeitos realizaram três esforços máximos com duração de 5 segundos e intervalo de 5 minutos $(19,20)$ entre cada contração. A CVM foi determinada pela média dos nove valores obtidos nos três dias de teste.

Em outros quatro dias, os sujeitos realizaram contrações submáximas em intensidades correspondentes a 5\%,10\%,15\% e 20\% da CVM até a exaustão. Essas contrações foram distribuídas randomicamente com razão de duas contrações por dia, separadas por um intervalo mínimo de 1 hora $(5,6,21,22)$. Entre cada dia de teste o intervalo foi de no mínimo $24 \mathrm{~h}$ e no máximo $48 \mathrm{~h}$. A exaustão foi definida como o abaixamento do tronco ou a variação maior do que $1 \mathrm{~kg}$ da intensidade da contração estipulada para a contração submáxima. Ao fim da contração submáxima o TRI foi registrado.

\section{Eletromiografia}

Depois de adequada preparação da pele (abrasão com lixa fina, limpeza com álcool e tricotomia), quatro pares de eletrodos de superfície descartáveis (Kendall Medi-Trace ${ }^{\circledR} 200$ ), de formato circular, monopolares (configuração bipolar), de $\mathrm{Ag} / \mathrm{AgCl}$ e com diâmetro de $1 \mathrm{~cm}$ foram posicionados bilateralmente sobre o músculo eretor da espinha $(23,24)$ nos níveis de L2-L3 (6 cm lateralmente) e L4-L5 (3 cm lateralmente), com distância intereletrodos de $3 \mathrm{~cm}$. Um eletrodo de referência foi posicionado no punho direito sobre o processo estiloide da ulna.

Os sinais EMG dos diferentes níveis vertebrais avaliados foram registrados por um eletromiógrafo equipado com um módulo de aquisição de sinais biológicos (Lynx ${ }^{\circledR}$ Tecnologia Eletrônica LTDA.) calibrado com ganho de 1.000 vezes, filtro passa-alta de $10 \mathrm{~Hz}$ e filtro passa-baixa de $500 \mathrm{~Hz}$, e por uma placa conversora analógico-digital (A/D) (CAD 1026, Lynx $^{\circledR}$ Tecnologia Eletrônica LTDA.) com faixa de entrada de -5 a +5 volts, resolução de 10 bits e modo comum de rejeição $>70 \mathrm{~dB}$. Também foi utilizado um software específico (AQDADOS 4, Lynx ${ }^{\circledR}$ Tecnologia Eletrônica LTDA.) por meio do qual a freqüência de amostragem foi calibrada em $1.000 \mathrm{~Hz}$.

O sinal EMG do músculo eretor da espinha e a força de tração exercida na célula de carga foram registrados em pacotes com duração de 5 segundos obtidos sem intervalo durante toda a contração submáxima.

\section{Tratamento dos dados}

Por meio de rotinas específicas, desenvolvidas em ambiente Matlab (Matlab 6.5, Mathworks ${ }^{\circledR}$ ), valores de frequência mediana (FM) foram obtidos para cada um dos pacotes de sinal EMG. Esses valores foram correlacionados com o TRI em cada uma das contrações submáximas utilizando a correlação linear de Pearson. Essa análise permitiu a identificação de coeficientes angulares, valores indicativos do grau de inclinação (slope) 
da reta gerada pela correlação de ambas as variáveis. A fadiga do músculo eretor da espinha foi determinada pela identificação de valores de slope negativos.

\section{Análise estatística}

A distribuição dos dados foi testada por meio do teste de Kolmogorov-Smirnov e a estatística paramétrica foi utilizada.

A comparação entre os TRI assim como a comparação entre os slopes da FM obtidos das diferentes intensidades de contração foi realizada utilizando a análise de variância (ANOVA). Quando necessário, o teste de post hoc Tukey foi utilizado.

As comparações entre os níveis de fadiga muscular de porções do músculo eretor da espinha localizadas em L2-L3 e L4-L5, em ambos os lados da coluna lombar, assim como as comparações entre os níveis de fadiga muscular de porções contralaterais de um mesmo nível vertebral do músculo eretor da espinha foram realizadas por meio do teste $T$ de Student para amostras indepentes.

Em todos os testes estatísticos os resultados foram considerados significantes, com $\mathrm{p} \leq 0,05$.

\section{Resultados}

O valor médio da CVM foi 40,6 \pm 9,6 kg. As médias dos TRI nas contrações submáximas foram de $134 \pm 42$ segundos em 5\%, $82 \pm 27$ segundos em 10\%, $72 \pm 27$ segundos em 15\% e $62 \pm 28$ segundos em $20 \%$, sendo encontradas diferenças significantes entre 5\% e 10\% (p = 0,04), 5\% e 15\% (p = 0,02) e também entre $5 \%$ e $20 \%(p=0,01)$.

Os valores médios de slope da FM em cada uma das intensidades de contração são apresentados na Tabela 1. Esses resultados demonstraram que a fadiga muscular foi identificada de forma significante bilateralmente em ambos os níveis vertebrais avaliados e em todas as intensidades de contração utilizadas.

A intensidade da contração não demonstrou efeito algum sobre o nível de fadiga do músculo eretor da espinha do lado direito nos níveis de L2-L3 ( $p=0,55)$ e L4-L5 ( $p=0,37)$ assim como do lado esquerdo nos níveis de L2-L3 ( $\mathrm{p}=0,46)$ e L4-L5 ( $\mathrm{p}=0,29)$.

Tabela 1 - Valores médios dos slopes de FM e seus respectivos dados estatísticos

(Continua)

\begin{tabular}{lcccc}
\hline \multicolumn{5}{c}{ Eretor da espinha direito } \\
\hline nível vertebral & \% CVM & slope da FM (Hz/s) & $\mathbf{r}^{2}$ & $\mathbf{p}$ \\
\hline \multirow{2}{*}{ L2-L3 } & 5 & -1.78 & 0.75 & 0.005 \\
& 10 & -2.02 & 0.79 & $<0.0001$ \\
& 15 & -1.82 & 0.64 & 0.04 \\
& 20 & -1.18 & 0.66 & 0.04 \\
\hline \multirow{2}{*}{ L4-L5 } & 5 & -3.43 & 0.90 & 0.0001 \\
& 10 & -4.47 & 0.89 & $<0.0001$ \\
& 15 & -4.41 & 0.89 & 0.0002 \\
& 20 & -5.25 & 0.91 & 0.0001
\end{tabular}


Tabela 1 - Valores médios dos slopes de FM e seus respectivos dados estatísticos

(Conclusão)

\begin{tabular}{lcccc}
\hline \multicolumn{5}{c}{ Eretor da espinha esquerdo } \\
\hline nível vertebral & \% CVM & slope da FM (Hz/s) & $\mathbf{r}^{\mathbf{2}}$ & $\mathbf{p}$ \\
\hline \multirow{2}{*}{ L2-L3 } & 5 & -1.81 & 0.69 & 0.04 \\
& 10 & -2.10 & 0.74 & 0.002 \\
& 15 & 2.06 & 0.68 & 0.02 \\
\hline L4-L5 & 20 & -2.44 & 0.67 & 0.04 \\
\hline 5 & -3.04 & 0.84 & 0.003 \\
& 10 & -3.82 & 0.90 & 0.0001 \\
& 15 & -3.34 & 0.85 & $<0.0001$ \\
& -5.20 & 0.89 & $<0.0001$ \\
\hline
\end{tabular}

Maiores níveis de fadiga muscular foram encontrados em L4-L5 nos músculos eretor da espinha direito $(5 \%: p=0,01 ; 10 \%: p=0,002 ; 15 \%: p=0,004 ; 20 \%: p=0,006)$ e esquerdo $(5 \%: p=0,04 ; 10 \%: p=0,01 ; 15 \%:$ $\mathrm{p}=0,03 ; 20 \%: \mathrm{p}=0,01)$ (Gráficos 1A e 1B respectivamente). Por outro lado, diferenças significantes não foram encontradas na comparação entre os níveis vertebrais de L2-L3 (5\%: $\mathrm{p}=0,77 ; 10 \%: \mathrm{p}=0,45 ; 15 \%$ : $\mathrm{p}=0,32 ; 20 \%: \mathrm{p}=0,07)$ e L4-L5 (5\%: $\mathrm{p}=0,63 ; 10 \%: \mathrm{p}=0,28 ; 15 \%: \mathrm{p}=0,14 ; 20 \%: \mathrm{p}=0,85)$ localizados do lado direito e esquerdo da coluna lombar (Gráficos 1C e 1D respectivamente).

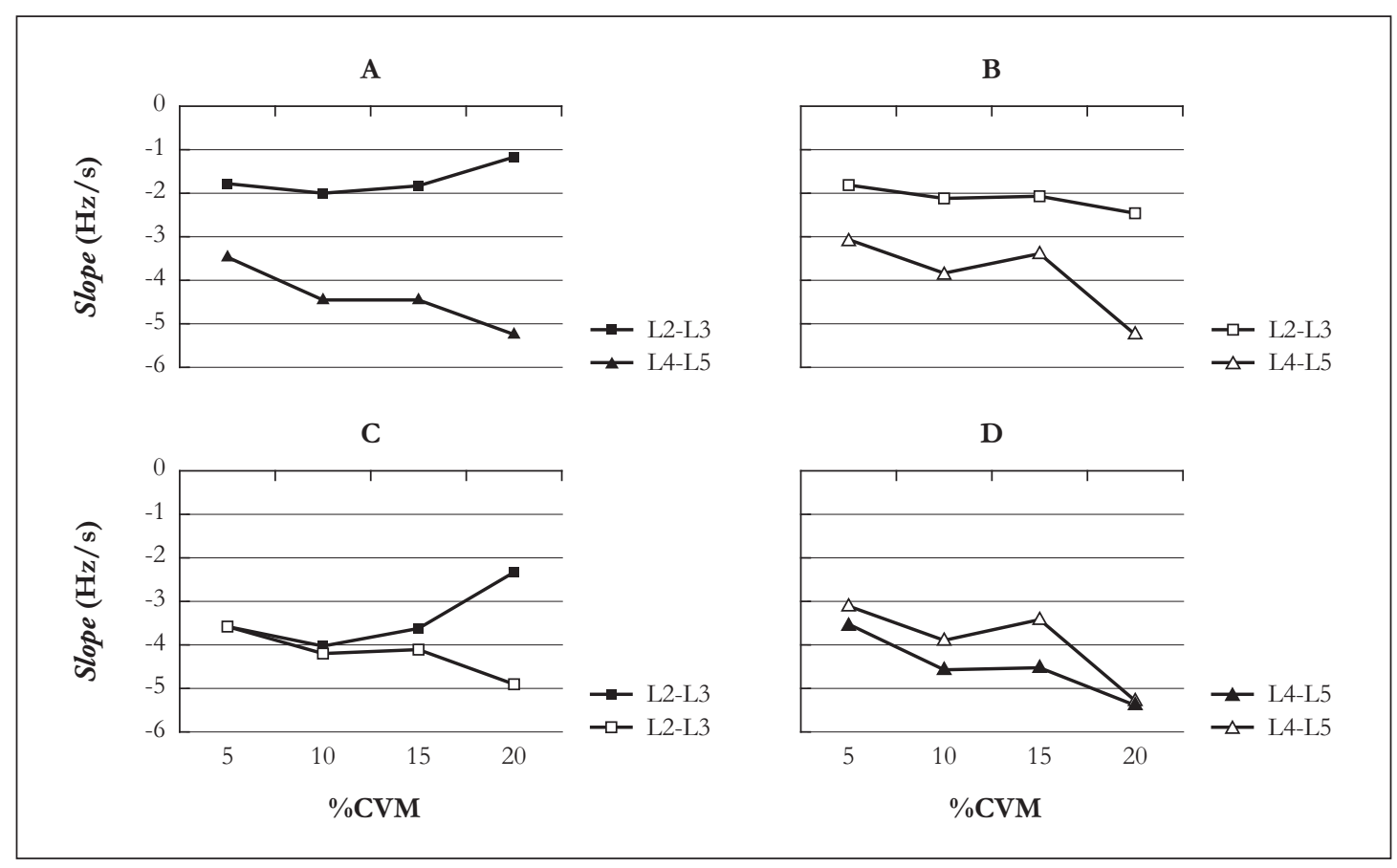

Gráfico 1 - Comparações entre os valores de slope da FM Lado Direito: ! L2-L3; \% L4-L5 / Lado Esquerdo: э L2-L3; + L4-L5. 


\section{Discussão}

Por meio de análises de correlação realizadas no presente estudo foi possível identificar valores de coeficiente de determinação elevados $\left(r^{2}>0,60\right)$ e significantes $(p \leq 0,05)$ em ambos os níveis vertebrais avaliados, demonstrando a existência de uma importante relação entre o declínio da FM e o TRI. Esse resultado também indica que o modelo de regressão linear dos valores de FM em função do tempo representa uma metodogia estatística adequada para a análise desta variável e, consequentemente, da fadiga muscular.

Esse resultado está de acordo com estudos prévios $(7,8)$, nos quais, em um teste similar ao realizado no presente estudo, também foram encontrados valores de coeficiente de determinação elevados $\left(\mathrm{r}^{2}>0,68\right)$, indicando que o TRI contribuiu com mais de $60 \%$ da variação da FM ao longo do tempo de realização do teste.

A ausência de uma relação direta entre o nível de fadiga e a intensidade da contração indica que contrações com intensidade entre 5\% e 20\% da CVM são responsáveis pela imposição de sobrecargas semelhantes à coluna lombar durante esforços isométricos. Em adição, a natureza altamente sinérgica dos músculos da coluna vertebral pode ter contribuído, por meio de uma estratégia de transferência de momentos de força entre estes músculos, assim como para músculos extensores do quadril (25), reduzindo a interferência da intensidade da contração sobre o nível de fadiga muscular. Esse fato foi confirmado pelo desconforto referido pelos sujeitos nos músculos posteriores do quadril e da coxa, como previamente foi reportado em estudos similares $(7,8,15,16)$.

Outra variável que pode ser introduzida nessa análise é a dominância manual dos sujeitos. Merletti et al. (26) e Sung et al. (27) demonstraram a existência de um efeito desta variável em alguns parâmetros EMG e verificaram que o músculo eretor da espinha contralateral à dominância manual dos sujeitos demonstrou uma tendência a menor fadiga, quando comparado com o músculo ipsilateral. De acordo com o autor, esse fato deve-se a um efeito de treinamento involuntário induzido ao músculo eretor da espinha por meio de AVD que são realizadas predominantemente com o membro superior dominante.

Particularmente no presente estudo, os nove sujeitos eram destros e, deste modo, considerando esses estudos, seria esperado maior nível de fadiga no músculo eretor da espinha direito. Contudo, em ambos os níveis vertebrais avaliados esse resultado não foi encontrado, indicando que na amostra estudada a dominância não afetou bilateralmente o condicionamento do músculo eretor da espinha.

Por outro lado, o maior nível de fadiga observado bilateralmente no nível de L4-L5 pode, de acordo com Sirca e Kostevc (28), ser atribuído a uma predominância de fibras do tipo II (glicolíticas/baixa resistência à fadiga) em porções mais inferiores da coluna lombar. Esse tipo de fibra caracteriza-se por uma importante tendência ao acúmulo de metabólitos que tem demonstrado ser fortemente correlacionada com o declínio da FM em consequência da fadiga muscular $(29,30)$.

\section{Conclusão}

O protocolo proposto foi efetivo para a identificação de forma objetiva da fadiga do músculo eretor da espinha. Os fatores intensidade de contração e lateralidade demonstraram não influenciar a fadiga muscular, enquanto que o nível de vertebral foi capaz de evidenciar diferenças funcionais entre regiões mais altas e mais baixas da coluna lombar, as quais demonstraram fadigar-se em intensidade diferente.

\section{Agradecimentos}

Agradecemos ao Conselho Nacional de Desenvolvimento Científico e Tecnológico (CNPq), número do auxílio: 130444/2004-9, à Coordenação de Aperfeiçoamento de Pessoal de Nível Superior (CAPES) e à Fundação para o Desenvolvimento da Unesp (Fundunesp). 


\section{Referências}

1. Dataprev, CAT. Estatísticas da previdência social. 2007. [acesso 20 dez. 2009]. Disponível em: http://www.mpas. gov.br/12.asp

2. Adams MA, Mannion AF, Dolan P. Personal risk factors for first-time low back pain. Spine. 1999;24(23):2497-505.

3. Dunn KM, Jordan K, Croft PR. Characterizing the course of low back pain: a latent class analysis. Am J Epidemiol. 2006;163(8):754-61.

4. Luoto S, Heliovaara MH, Hurri H, Alaranta H. Static back endurance and the risk of low-back pain. Clin Biomech. 1995;10:323-4.

5. Lariviére C, Gagnon D, Gravel D, Bertrand AA. The assessment of back muscle capacity using intermittent static contractions. Part I - Validity and reliability of electromyographic indices of fatigue. J Electromyogr Kinesiol. 2008;18(6):1006-19.

6. Lariviére C, Gagnon D, Gravel D, Bertrand AA. The assessment of back muscle capacity using intermittent static contractions. Part II: validity and reliability of biomechanical correlates of muscle fatigue.J Electromyogr Kinesiol. 2008;18(6):1020-31.

7. Mannion AF, Dolan P. Electromyographic median frequency changes during isometric contraction of the back extensors to fatigue. Spine. 1994;19(11):1223-9.

8. Clark BC, Manini TM, Mayer JM, Ploutz-Snyder LL, Graves JE. Electromyographic activity of the lumbar and hip extensors during dynamic trunk extension exercise. Arch Phys Med Rehabil. 2002;83(11):1547-52.

9. Ng JK-F, Richardson CA, Jull GA. Electromyographic amplitude and frequency changes in the iliocostalis lumborum and multifidus muscles during a trunk holding test. Phys Ther. 1997;77(9):954-61.

10. Coorevits P, Danneels L, Cambier D, Ramon H, Druyts H, Karlsson JS, et al. Test-retest reliability of wavelet - and Fourier based EMG (instantaneous) median frequencies in the evaluation of back and hip muscle fatigue during isometric back extensions. J Electromyogr Kinesiol. 2008;18:798-806.

11. Yoshitake Y, Ue H, Miyazaki M, Moritani T. Assessment of lower-back muscle fatigue using electromyography, mecanomyography, and near-infrared spectroscopy. Eur J Appl Physiol. 2001;84(3):174-9.

12. Elfving B, Dedering A, Németh G. Lumbar muscle fatigue and recovery in patients with long-term low-back trouble-electromyography and health-related factors. Clin Biomech. 2003;18(7):619-30.

13. Biering-Sorensen F. Physical measurements as risk indicators for low back trouble over a one-year period. Spine. 1984;9(2):106-19.

14. Sung PS, Lammers AR, Danial P. Different parts of erector spinae muscle fatigability in subjects with and without low back pain. Spine. 2009;9(2):115-20.

15. Sparto PJ, Parnianpour M, Reinsel TE, Simon S. Spectral and temporal responses of trunk extensor electromyography to an isometric endurance test. Spine. 1997;22(4):418-25.

16. Süüden E, Ereline J, Gapeyeva H, Pääsuke M. Low back muscle fatigue during Sorensen endurance test in patients with chronic low back pain: relationship between electromyographic spectral compression and anthropometric characteristics. Electromyogr Clin Neurophysiol. 2008;48(34):185-92.

17. Hides JA, Richardson CA, Jull GA. Multifidus muscle recovery is not automatic after resolution of acute, firstepisode low back pain. Spine. 1996;21(23):2763-9.

18. Barbosa FSS, Gonçalves M. Comparação entre protocolos de exaustão e de 30 segundos utilizados na avaliação da fadiga eletromiográfica dos músculos eretores da espinha. Rev Bras Fisioter. 2005;9(3):77-83. 
19. Billeter R, Hoppeler H. Muscular basis of strength. In: Komi P. Strength and power in sport. Oxford: Blackwell Scientific Publications; 1996. p. 39-63.

20. Zhang ZG, Liu HT, Chan SC, Luk KD, Hu Y. Time-dependent power spectral density estimation of surface electromyography during isometric muscle contraction: methods and comparisons. J Electromyogr Kinesiol. Electromyogr Kinesiol. 2008;19:181-92.

21. Ebenbichler G, Kollmitzer J, Quittan M, Uhl F, Kirtley C, Fialka V. EMG fatigue patterns accompanying isometric fatiguing knee-extensions are different in mono- and bi-articular muscles. Electroencephalogr Clin Neurophysiol. 1998;109(3):256-62.

22. Masuda K, Masuda T, Sadoyama T, Inaki M, Katsuta S. Changes in surface EMG parameters during static and dynamic fatiguing contractions. J Electromyogr Kinesiol. 1999;9(1):39-46.

23. Tsuboi T, Satou T, Egawa K, Izumi Y, Miyazaki M. Spectral analysis of electromyogram in lumbar muscles: fatigue induced endurance contraction. Eur J Appl Physiol Occup Physiol. 1994;69(4):361-6.

24. Herrmann CM, Madigan ML, Davidson BS, Granata KP. Effect of lumbar extensor fatigue on paraspinal muscle reflexes. J Electromyogr Kinesiol. 2006;16(6):637-41.

25. Vleeming A, Poolgoudzwaard AL, Stoeckart R, Vanwingerden JP, Snijders CJ. The posterior layer of the thoracolumbar fascia: its function in load transfer from spine to legs. Spine. 1995;20(7):753-8.

26. Merletti R, de Luca CJ, Sathyan D. Electrically evoked myoelectric signals in back muscles: effect of side dominance. J Appl Physiol. 1994;77(5):2104-14.

27. Sung PS, Spratt KF, Wilder DG. A possible methodological flaw in comparing dominant and nondominant sided lumbar spine muscle responses without simultaneously considering hand dominance. Spine. 2004;29(17):1914-22.

28. Sirca A, Kostevc V. The fibre type composition of thoracic and lumbar paravertebral muscles in man. J Anat. 1985;141:131-7.

29. Laurent D, Portero P, Goubel F, Rossi A. Electromyogram spectrum changes during sustained contraction related to proton and deprotonated inorganic phosphate accumulation: a 31P nuclear magnetic resonance study on human calf muscles. Eur J Appl Physiol. 1993;66:263-8.

30. Barbosa FSS, Gonçalves M. A proposta biomecânica para a avaliação de sobrecarga na coluna lombar: efeito de diferentes variáveis demográficas na fadiga muscular. Acta Ortop Brás. 2007;15(3):132-7.

Recebido: 26/01/2009

Received: 01/26/2009

Aprovado: $14 / 06 / 2010$

Approved: 06/14/2010 